\title{
El rol del Estado en los países en desarrollo en la teoría evolucionista de Carlota Pérez. El caso del sector de software de Córdoba, Argentina*
}

\author{
Carina Mariana Borrastero **
}

\section{RESUMEN}

Proponemos un análisis crítico de la concepción del Estado en la teoría evolucionista de Carlota Pérez. Sugerimos que la autora mantiene una concepción restringida de los roles del Estado en los procesos de desarrollo, que dificulta la comprensión de los vínculos que dicho actor establece con los agentes privados. A partir de esta hipótesis analizamos la intervención estatal en el desarrollo del sector de software y servicios informáticos de Córdoba, Argentina, que muestra actualmente altos indicadores de desempeño significativamente marcados, desde nuestro punto de vista, por las características de dicha intervención. Sostenemos, finalmente, que ésta presenta rasgos que ponen en cuestión la concepción del Estado de Pérez.

Palabras clave: rol del Estado, articulación pública-privada, países en desarrollo, teoría evolucionista de Carlota Pérez, sector de software y servicios informáticos de la ciudad de Córdoba, Argentina.

Clasificación JEL: O10

\begin{abstract}
We propose a critical analysis of the conception of the State in the evolutionary theory of Carlota Pérez. We suggest that the author maintains a narrow conception of the roles of the State in development processes, that hinders the understanding of the links that this actor establishes with private agents. From this hypothesis we analyze the nature of State intervention in the development of the Software and Information Technology sector of the city of Cordoba, Argentina, currently showing high performance indicators which, from our point of view, are significantly determined by the characteristics of such intervention. We sustain that said intervention has features that call into question the conception of the State by the author.
\end{abstract}

Keywords: role of the State, public-private links, developing countries, evolutionary theory of Carlota Pérez, Software and IT Services Sector in the city of Córdoba, Argentina. JEL classification: $\mathrm{O} 10$

* Fecha de recepción: 27/09/2011. Fecha de aprobación final: 26/05/2012.

** Instituto de Altos Estudios Sociales de la Universidad Nacional de San Martín. Buenos Aires, Argentina. Consejo Nacional de Investigaciones Científicas y Técnicas (Conicet), Argentina. Universidad Nacional de Córdoba, Argentina. Candidata a doctora en Ciencias Sociales por la Universidad de Buenos Aires, Argentina. Correo electrónico: carinaborrastero@conicet.gov.ar 


\section{INTRODUCCIÓN}

El objetivo del presente artículo es analizar desde una perspectiva crítica la concepción de Carlota Pérez sobre el rol del Estado en los procesos de cambio estructural y desarrollo. En particular, se analizarán las implicaciones de su concepción para los países en desarrollo (PED), en contraste con algunas evidencias acerca de la intervención estatal que surgen del análisis del desarrollo del sector de software y servicios informáticos (SSI) de la ciudad de Córdoba, Argentina. El interés particular por dicha autora reside principalmente en su visión histórica de la dinámica y de los actores de la innovación, a partir de la cual resulta posible analizar la relación entre los cambios mundiales de paradigmas tecnoeconómicos y el impacto de esos cambios en las economías nacionales y regionales. Por otro lado, se constata la amplia recepción de su trabajo entre otros autores importantes que se ocupan de los problemas de la innovación y el desarrollo, en general, y del rol del Estado, en particular, al interior de estos procesos. ${ }^{1}$ Ello sugiere la necesidad de comprender en profundidad la perspectiva de Pérez y analizar sus implicaciones específicas para los PED. En este sentido, el análisis empírico de la intervención estatal en el sector donde se produce el "factor clave" del actual paradigma, en una región de Argentina, puede ayudar a distinguir el alcance de la propuesta de esta investigadora en relación con la cuestión del Estado. Como hipótesis de trabajo general, se sostiene que la evolución reciente del sector de SSI de Córdoba ha estado profundamente marcada por la intervención estatal y se sugiere que los resultados del análisis de dicho proceso no necesariamente condicen con la concepción de Pérez sobre el rol estatal en el desarrollo.

La sección I del artículo describe analíticamente la perspectiva general de Carlota Pérez; en la sección II se analiza específicamente su concepción sobre el papel del Estado en los procesos de desarrollo y sus implicaciones para los PED, y se introducen algunas nociones provenientes de la sociología del desarro1lo, útiles como variables operativas para analizar la intervención estatal en procesos de desarrollo. En la sección III, y a modo de conclusión, se presenta un análisis preliminar e ilustrativo de la incidencia de la intervención estatal en la evolución reciente del sector de SSI de Córdoba y se precisan las hipótesis con respecto a la fecundidad de la perspectiva de Pérez para analizar este tipo de fenómenos.

\footnotetext{
${ }^{1}$ Véase, por ejemplo, Castells (1996 y 1988a), Boyer (1999) y Jessop (2000).
} 


\section{Paradigma tecnoeconómico Y CAMbio eSTRUCTURAL EN La teoría eVolucionista de CARlota Pérez}

Carlota Pérez propone una teoría de los ciclos largos de la economía basada en el concepto de paradigmas tecnoeconómicos (PTE). Partiendo de ello, analiza los indicadores de cambios de paradigma que definen periodos históricos determinados por las formas predominantes de producción económica.

El planteamiento nuclear de su teoría es que la evolución del sistema en su conjunto está guiada por los cambios de paradigma:

Aquí proponemos considerar al sistema capitalista como una estructura única sumamente compleja, cuyos subsistemas tienen distintos ritmos de evolución. Simplificando, podemos asumir dos subsistemas fundamentales: el tecnoeconómico por un lado y el socioinstitucional por el otro, donde el primero tiene una capacidad de respuesta más rápida que el segundo. Los ciclos largos serían fases sucesivas de evolución del sistema en su conjunto o, según la terminología propuesta, sucesivos modos de crecimiento. El motor básico de la dinámica del sistema sería la búsqueda de la ganancia como fuerza generadora de innovaciones en la esfera productiva, definidas éstas en su sentido más amplio como formas de elevar la productividad y las ganancias esperadas de las inversiones nuevas (Pérez, 1985, p. 5)

La autora postula que las ondas largas de Kondratieff no son un fenómeno estrictamente económico, sino una manifestación medible en términos económicos del comportamiento total del sistema socioeconómico e institucional nacional e internacional, que durante ciertos periodos resulta armónico y en otros no (Pérez, 1985). Cada uno de los ciclos identificables en esta evolución se asimila a un modo de desarrollo, concebido como un patrón general de crecimiento basado en la aceptación de un conjunto de mecanismos sociales e institucionales, tanto en el plano nacional como en el internacional, cuya acción influye en la operación de todos los mercados (Pérez, 1985). La causa de la forma cíclica del proceso de evolución

[...] residiría en el hecho de que, para brindar su pleno potencial de crecimiento, cada una de las revoluciones -o cambios de "paradigma tecnoeconómico", como los llamaremos aquí- requiere una reestructuración del marco socioinstitucional, tanto en el plano nacional como en el internacional. Las transformaciones 
sociales e institucionales resultantes determinan entonces la forma general del crecimiento económico o el "modo de crecimiento" de la siguiente onda larga. De esta manera, una onda Kondratieff se define como el auge y caída de un modo de crecimiento y cada crisis como la ardua transición de un modo de crecimiento al otro (Pérez, 1985, p. 3).

Lo que orienta esta dinámica de transformación son sucesivos patrones tecnológicos, que implican sucesivos saltos cualitativos en el nivel de productividad general alcanzable, lo cual supone un enorme potencial de creación de riqueza. Dichos saltos se basan en la aparición de constelaciones de innovaciones interrelacionadas, tanto en el terreno estrictamente técnico como en el organizativo, impulsadas por la búsqueda de ganancias. De esta manera, en el modelo de esa autora, la historia es una sucesión de modos de desarrollo determinados por distintos PTE, que surgen a partir de revoluciones tecnológicas (RT), motivadas por la búsqueda de ganancias.

Las RT son conjuntos de avances tecnológicos radicales que emergen juntos en un momento dado, formando una constelación de tecnologías y sistemas tecnológicos interdependientes capaz de transformar las maneras de producir de toda la economía (Pérez, 2009a). Cada RT se basa en una modificación radical y duradera de la estructura de costos relativos de la totalidad de los insumos productivos, que impulsa a algunos a la baja y a otros al alza durante periodos más o menos largos, permitiendo una importante capacidad de previsión. La mayor eficiencia a menor costo es posibilitada por la aparición (o difusión) de un factor clave, un insumo estratégico que, por su capacidad de influir sobre el comportamiento de la estructura de costos relativos, produce el salto radical de productividad. ${ }^{2}$ Dichas cualidades las poseen en la actualidad, según Pérez, el microchip y el software, los insumos básicos de la microelectrónica y la informática. De modo que el factor clave permite la propagación de la RT a todas las ramas de la producción y a escala mundial, es decir, a cualquier tipo de sociedad. Una RT no se trata únicamente, por lo tanto, de un conjunto de nuevas técnicas, productos o procesos, sino de transformaciones "interdependientes en lo técnico,

\footnotetext{
${ }^{2}$ Para ello su costo relativo debe ser perceptiblemente bajo y con tendencias decrecientes, su oferta ilimitada, su potencial de uso para diversos propósitos productivos debe aparecer como universal en forma evidente y debe constituir la base de un sistema de innovaciones técnicas y organizativas reconocidas como capaces de modificar el perfil y reducir los costos de equipamiento, mano de obra y productos.
} 
lo organizativo, lo gerencial y lo social. Es precisamente porque todo lo penetra" (Pérez, 1998, p. 5, cursivas del original).

Una vez que la RT ha surgido, las cualidades del factor clave se difunden y conforman de este modo un tipo ideal de organización productiva, que define las combinaciones más eficientes y menos costosas para la producción durante un período dado y funciona así como norma implícita orientadora de las decisiones de inversión y de innovación tecnológica (Pérez, 1986). Este patrón productivo que cambia con cada RT es lo que la autora denomina paradigma tecnoeconómico. Pero, al igual que la RT, el PTE no está en realidad asociado únicamente a cambios en la esfera productiva, sino que la trasciende involucrando la totalidad del sistema. En efecto, las transformaciones radicales suscitadas en el ámbito técnico provocan un desajuste estructural profundo entre el subsistema tecnoeconómico y el subsistema socioinstitucional, debido a que, cuando los cambios en los modos de producir son tan radicales, se generan problemas de status quo que requieren una adaptación del marco socioinstitucional vigente (Pérez, 2009a):

Ese proceso de innovación social y política es naturalmente prolongado y profundamente conflictivo. [...] Hasta que no se restablezca la coherencia estructural, logrando un contexto socioinstitucional favorecedor del nuevo potencial que trata de abrirse paso en la esfera tecnoeconómica, no es posible relanzar la producción sobre un camino de expansión duradera (Pérez, 1986, p. 8).

Por ello, sugiere, "lo que un paradigma brinda es un conjunto coherente de principios que constituyen la forma más avanzada, eficaz y eficiente de organización e interrelación que posee la sociedad en un período dado" (Pérez, 1998, p. 14). El principio organizativo básico del PTE dominante en la actualidad, basado en la microelectrónica y la informática, es la organización en red. Las redes son conjuntos de instancias organizativas descentralizadas pero interactivas, que pueden estar o no bajo coordinación central. Cada nodo de la red goza de autonomía local en la toma de decisiones y la ejecución de acciones, y la coordinación entre ellos es de tipo adaptativo, basada en un nuevo liderazgo que la autora reseñada denomina "gerencia estratégica dinámica" y que infundiría a la red un dinamismo muy superior al de las estructuras centralizadas. La característica distintiva de la organización en red es la flexibilidad, su capacidad de adaptación a una variedad de contextos y a los cambios internos de contextos determinados. En este sentido, las redes son abiertas y globalizadas y pueden ponerse al servicio 
de objetivos innumerables y radicalmente diversos. La eficacia de este principio organizativo pone en cuestión, según la investigadora, las formas de organización burocráticas, centralizadas y jerárquicas que configuraron el paradigma de la producción en masa (Pérez, 1986). En efecto, para ella,

[...] la clave de la efectividad de las organizaciones en red es el consenso. Las redes no funcionan si no hay acuerdo sobre la visión perseguida. La vieja imposición de la autoridad o de la decisión de la mayoría no funciona: obedecer, estando en desacuerdo, es factible, pero es imposible ser creativo, sin estar verdaderamente compenetrado con el rumbo común (Pérez, 1985, p. 35).

Según su teoría, el principio de la red se extiende a todos los ámbitos transformándose en un "sentido común" generalizado para casi todas las actividades, debido a lo cual todos los tipos de organización tienden a estructurarse del mismo modo (Pérez, 1997, p. 8). De esta manera, se entrelazan lo técnico, lo organizativo y lo institucional, configurando al PTE vigente como óptimo universal de eficiencia en su época (Pérez, 1998).

Ahora bien, si el grado de sinergia social es tal, ¿qué es lo que da lugar al cambio de PTE, según la teoría de Pérez? ¿Cuándo están dadas las condiciones para que los parámetros sistémicos se transformen? El momento del cambio llega cuando se ha agotado el potencial técnico-económico del paradigma anterior, esto es, cuando los beneficios del insumo clave en términos de costos relativos se han difundido ya a toda la economía y han permanecido durante un tiempo considerable. Esto es lo que ocurre aproximadamente cada 50 años.

Lo verdaderamente nuevo, entonces, no es el mero hecho técnico. La ruptura se produce cuando se entrelazan lo técnico y lo económico a través de una dramática reducción del costo relativo del insumo o conjunto de insumos clave, como consecuencia de una serie de eventos, algunos fortuitos y otros motivados, incluyendo una constelación de innovaciones técnicas y organizativas radicales (Pérez, 1986, p. 5).

Es este proceso de interacción y retroalimentación entre la esfera económica y la tecnológica el que conduce al surgimiento de nuevos elementos técnicos (Pérez, 1985). De allí que, para Pérez, "the meaningful space where technical change needs to be studied is that of innovation, at the convergence of technology, 
the economy and the socio-institutional context. That space is essentially dynamic" (Pérez, 2009a, p. 3, resaltado en el original).

Establece así una distinción entre los conceptos de cambio técnico e innovación, que le es útil para enfatizar la dimensión económica de esta última, cuya definición toma de Schumpeter. Al respecto, señala que

[...] el proceso de avance tecnológico, en términos de producción de conocimientos e invenciones es un proceso relativamente autónomo, pero la innovación -es decir, la aplicación y difusión de técnicas específicas en la esfera productiva- está fuertemente determinada por las condiciones sociales y las decisiones tomadas en aras del beneficio económico (Pérez, 1985, p. 5).

De este modo, la incidencia de los factores económicos en la evolución general del sistema tiene una relevancia sustancial en su modelo, al igual que en otros modelos evolucionistas, ${ }^{3}$ pero adquiere aquí matices específicos: las fuerzas económicas no sólo actúan en la fijación de la dirección del cambio, ${ }^{4}$ a través de las condiciones de oferta y demanda que impone el mercado, sino que se encuentran en el origen de la emergencia de las novedades técnicas al ser-como ya hemos apreciado en las citas anteriores- la búsqueda de la ganancia el motor principal del cambio, y por el hecho de que la difusión del insumo clave surgido en el campo tecnológico permite una reducción drástica de los costos globales de producción. Podemos decir entonces que el paradigma es tecnoeconómico porque el proceso de innovación genera, en el mismo movimiento, formas novedosas de conocimiento y riqueza.

¿Qué rol específico atribuye Pérez a las instituciones en la evolución del sistema? En primer lugar, es necesario advertir que no hemos encontrado una definición explícita de "instituciones" en la literatura revisada de esta autora. No obstante, no es difícil suponer, a partir de lo ya dicho, que la concepción de Pérez involucra, por un lado, a las organizaciones que no forman parte del mercado (su función no es la de producir bienes y servicios), pero que interactúan en forma permanente con las empresas e inciden de ese modo en la forma y los resultados del proceso de producción. En este marco incluye, principalmente, al Estado, a las instituciones del sistema científico-tecnológico y a las instituciones financieras. Por otro lado, involucra a los patrones de interacción entre distintas instancias del

\footnotetext{
${ }^{3}$ Véase Nelson y Winter (1982), y Dosi, Pavitt y Soete (1990).

${ }^{4}$ Véase, por ejemplo, Dosi (2003).
} 
sistema. ${ }^{5}$ En términos abarcadores y en innumerables pasajes, la autora analizada denomina a todas estas instancias del sistema -organizativas y normativas- "instituciones", "factores sociales", "fuerzas sociales", etcétera. Entendidas de esta manera, las instituciones ocupan un lugar de relevancia al interior del modelo: no sólo determinan la dirección del cambio técnico sino que son la instancia capaz de fijar la nueva configuración sistémica, en su globalidad, a partir del proceso de innovación adaptativa al cambio tecnoeconómico del que hablamos antes. Es decir, por un lado, "el cambio técnico puede ser acelerado o frenado por factores sociales” (Pérez, 1985, p. 5), pero, además,

la forma definitiva que tomará la nueva estructura dentro del amplio rango de alternativas abiertas, así como el tiempo que tome la transformación requerida para dar lugar a un nuevo ascenso, dependerá en última instancia de los intereses, las acciones, la lucidez y el poder relativo de las fuerzas sociales en juego (Pérez, 1985, p. 6, cursivas del original).

Entonces, el cambio se origina en el subsistema tecnoeconómico y el socioinstitucional debe reacoplarse a él, produciendo a su vez innovaciones de tipo adaptativo en su propio ámbito. Por un lado, la preeminencia de la esfera tecnoeconómica sobre la socioinstitucional es evidente, debido al mayor dinamismo de la primera. Por otro lado, se atribuye a las instituciones, de por sí más inertes por su apego a las prácticas ya ineficaces del paradigma anterior (Pérez, 2004), un rol definitorio del rumbo del sistema en su conjunto una vez que la revolución tecnológica se ha producido, impulsada por el afán de beneficios. ${ }^{6} \mathrm{De}$ esta manera, Pérez introduce una fuerte determinación económica para explicar el proceso de innovación que da origen al cambio de paradigma y abre así oportunidades de desarrollo.

\footnotetext{
${ }^{5}$ Para llegar a una noción más concreta de la idea de "institución" de Pérez, recuperamos algunos de los ejemplos de "innovaciones institucionales" a las que se refiere cuando explica el cambio de paradigma operado en la postguerra: a) en lo nacional, intervención estatal masiva en la economía (modelo keynesiano), suministro de estadísticas nacionales, sistemas de educación y de salud de masas, y otros; b) en lo internacional, papel hegemónico de EU, Bretton Woods, FMI, ONU, disolución gradual de los imperios coloniales, etc. (Pérez, 1998). Dentro del paradigma actual, la innovación institucional de mayor relevancia es la organización en red (véase adelante). La concepción de "institución" de esta autora está contenida en varios pasajes que nos sirven para ilustrar, en realidad, otros argumentos, centrados más bien en su papel explicativo dentro del modelo. Esto sucede, como dijimos, porque no hemos detectado en la bibliografía revisada una definición explícita que pueda ser reproducida en forma literal.

${ }^{6}$ Recordemos que la revolución es de carácter irreversible para Carlota Pérez.
} 
La consecuencia sistémica más vasta derivada del cambio de paradigma, que implica la difusión de una nueva racionalidad técnico-económica, es lo que esa autora denomina cambio estructural: la construcción y generalización de un tejido productivo diferente e impulsado por el crecimiento de ramas productivas distintas de las que prevalecieron en el periodo de auge del PTE anterior (Pérez, 1986). El cambio estructural es un proceso que modifica sensible y gradualmente la totalidad de los aspectos determinantes del sistema productivo (Pérez, 1985). Este tipo de modificaciones de gran profundidad afectan significativamente la estabilidad de los mercados, lo que exige transformaciones institucionales de un alcance equivalente que logren reencauzar el crecimiento a partir de los nuevos parámetros (Pérez, 1985). Los efectos socioeconómicos del cambio estructural son para esta investigadora profundamente conflictivos: obsolescencia de la antigua estructura de calificaciones valoradas económicamente, desempleo, destrucción de los medios de subsistencia de muchos, dislocación geográfica de las actividades y las personas, entre otros trastornos de envergadura, sumados a la ineficacia de las recetas gubernamentales aplicadas históricamente. La insistencia en la implementación de medidas ya desactualizadas puede, incluso, agravar la situación (Pérez, 2004).

Dado que los cambios de paradigma se producen de forma revolucionaria, el desarrollo se presenta también a grandes oleadas (great surges of development) (Pérez, 2009b). Se trata de un proceso de acumulación de capacidades tecnológicas y sociales, orientado al aprovechamiento de "ventanas de oportunidad" sucesivas y distintas que se abren con cada revolución tecnológica (Pérez, 2001, p. 1). En esta dirección, enfatiza que

la historia nos ha mostrado que los procesos exitosos e irreversibles de desarrollo siempre han sido procesos masivos de adquisición de capacidades tecnológicas a nivel social. [...] Son los únicos que garantizan que los esfuerzos de desarrollo sean no sólo exitosos sino sobre todo irreversibles. Ese vasto proceso de apropiación fue lo que hicieron los coreanos, los cuatros tigres asiáticos y los japoneses. Esto nos indica que la tecnología tiene que ser el núcleo dinámico de las estrategias de desarrollo. Sin dominar la tecnología, es posible el crecimiento, pero no es posible el desarrollo (Pérez, 2000, p. 13)

Esta autora introduce aquí una diferencia entre crecimiento y desarrollo. El crecimiento es un proceso económico en sentido estricto, analizable a través de instrumentos de medición del producto, como el PIB, y otros agregados 
económicos (Pérez, 2009a) que no implican, para ella, transformaciones significativas de orden socioinstitucional:

Great surges of development, by contrast, represent the gradual integral transformation of both the techno-economic and the socio-institutional spheres of the social system, through the assimilation of each major cluster of technical change. A great surge is thus defined as the process by which a technological revolution and its techno-economic paradigm propagate across the economy, leading to structural changes in production, distribution, communication and consumption, as well as to profound and qualitative social changes. Society, in turn, influences the path taken by the revolution. In other words, the concept stretches far beyond the economy, to encompass societal -even cultural-change (Pérez, 2004, p. 9).

Como puede verse, el proceso de desarrollo no implica únicamente a la instancia de la producción, que involucra el modo de generación de la riqueza (el modo de crecimiento), sino también a la instancia de distribución, cuya dinámica determina las posibilidades reales de elevación de los niveles de bienestar del conjunto de los agrupamientos sociales. De todos modos, es necesario aclarar que una revisión en profundidad del problema de la distribución está ausente en la perspectiva de Pérez. En términos muy generales, explica que

una vez establecido un modo de crecimiento adecuado, éste tiende a moldear, regular y determinar las formas principales de explotación del nuevo potencial tecnológico. Y, dado que un salto cuántico [sic] en productividad implica un salto cuántico en la capacidad de generación de riqueza, su aprovechamiento incluye, entre los resultados posibles, la elevación del nivel de vida de la mayoría de la población. [...] Cada transición, entonces, al exigir una reestructuración radical, reabre la cuestión de las perspectivas de desarrollo de los diversos países y de la mejor o peor distribución de los beneficios del crecimiento futuro entre los diversos grupos sociales, regiones y países (Pérez, 1985, p. 14).

¿De qué depende, para Pérez, la forma de distribución de los beneficios surgidos del nuevo paradigma? Al respecto, señala:

El crecimiento acelerado y una mayor equidad no van necesariamente juntos. Cada transición es una encrucijada histórica durante la cual las fuerzas sociales y políticas presentan sus programas y viven sus confrontaciones, entran en con- 
flicto o en procesos de consenso, para imponer por la fuerza o construir colectivamente el marco socioinstitucional específico que guiará la forma de crecimiento durante las décadas siguientes. El desenlace de este complejo proceso depende del poder relativo y de la lucidez de las fuerzas sociales en juego (Pérez, 1997, p. 4).

Quiere decir esto que el desarrollo, tal como lo comprende, no está garantizado por el cambio tecnoeconómico sino que constituye un proceso abierto cuyo resultado depende de la dinámica socioinstitucional del sistema una vez que un nuevo paradigma se ha instalado. La conjunción de crecimiento y equidad depende, en última instancia, de las relaciones de poder entre las "fuerzas sociales" (Pérez, 1997, p. 4).

En particular, esta investigadora se refiere a las implicaciones de tal concepción del desarrollo para pensar la situación y el potencial de los países en desarrollo. Para ella, el cambio de PTE es un fenómeno global, pero el cambio estructural y el desarrollo son de carácter nacional: dependen de la forma que adquieren, en cada país, los cambios económicos y sociales asociados al nuevo paradigma. Como ya vimos, considera que cada cambio de PTE abre una ventana de oportunidad que puede o no ser aprovechada por los PED. A través de ella puede producirse el "salto en el desarrollo" (Pérez, 2001, p. 4). Aquí, la expresión "salto" tiene un sentido literal: en la visión de Pérez, cada ventana ofrece a los PED la oportunidad de saltarse las etapas del desarrollo que ya han transitado los países desarrollados y alcanzar así los mismos niveles tecnológicos y socioeconómicos. Dicho salto, por lo general, implica la asunción de los parámetros tecnológicos que una vez introducidos en los países centrales se difunden a los periféricos $^{7}$ (Pérez, 2001, p. 10). Pero no implica la adopción de tecnologías ya maduras, sino la necesidad de impulsar en lo local el desarrollo del conocimiento y los mercados vinculados a las nuevas. El desarrollo nacional está entonces

\footnotetext{
${ }^{7}$ Es por ello que, para Carlota Pérez, los PED persiguen un "blanco móvil”, que no sólo avanza sino que cambia de dirección aproximadamente cada cincuenta años: cuando las tecnologías asociadas al PTE en implementación se difunden a la periferia, en el centro ya se está gestando una nueva oleada innovadora. Al respecto, dice: "Gran parte del aprendizaje tecnológico es gradual e incremental. Sin embargo, no hay ninguna progresión inevitable hacia una frontera cada vez más lejana y siempre inalcanzable; existen importantes discontinuidades que se convierten en ventanas por las que los recién llegados pueden saltar adelante. Estas oportunidades se dan en forma de revoluciones tecnológicas e implican fuertes cambios de dirección en el avance tecnológico" (Pérez, 2001, p. 5, cursivas del original).
} 
fuertemente ligado a la difusión del factor clave en la economía y al manejo del conocimiento asociado a éste, que permitan un cambio estructural positivo.

El aprovechamiento del potencial tecnológico que conlleva el cambio de PTE define la nueva situación de cada país en dos aspectos de relevancia: "Históricamente, cada transición ha modificado tanto las condiciones internas de las diversas capas y grupos sociales en cada país como la posición relativa de los países en la generación y distribución de la producción mundial" (Pérez, 1986, p. 7).

\section{EL ROL DEL ESTADO EN EL DESARROLLO. IMPLICACIONES PARA LOS PED}

\section{Crítica de la concepción del Estado de Carlota Pérez}

Si en la perspectiva de la autora reseñada el cambio estructural y el desarrollo son fenómenos nacionales, entendemos entonces que el papel del Estado en estos procesos debería constituir uno de los principales tópicos de reflexión teórica. Sin embargo, notamos que ni la naturaleza del Estado ni su rol sistémico aparecen como núcleos problemáticos en su análisis $\mathrm{y}$, por lo tanto, no son objeto de una reflexión conceptual sistemática.

En primer lugar, resulta llamativo que los sitios en que Pérez reflexiona sobre el Estado son casi exclusivamente los dedicados a la recomendación de políticas para los PED. Conceptualiza, pero en un sentido instrumental, o pragmático, y en términos normativos. En general, introduce elementos de la cuestión cuando piensa los aspectos institucionales de los procesos de cambio. Señala, por ejemplo:

La apertura a la competencia internacional, los programas de ajuste macroeconómico y las privatizaciones han sido, en la práctica, un modo efectivo de desmantelar las estructuras que presidieron el proceso de industrialización por sustitución de importaciones. Ese marco institucional, sin duda exitoso bajo el paradigma anterior, es ahora obsoleto y su mantenimiento nefasto. Esos cambios básicos del contexto nacional son precisamente los que han servido de acicate para que la empresa privada, antes protegida, descubra por sí misma las transformaciones que han estado ocurriendo en el mercado mundial y se decida a emprender su propia modernización (Pérez, 1998, p. 13). 
De esta manera, se refiere a los cambios que en su visión es necesario introducir en el Estado cuando critica los modos tradicionales de entender la cuestión:

A estas alturas se hace evidente que la dicotomía mercado vs. Estado es inadecuada. Aunque la magnitud y la complejidad de la tarea requieren un Estado fuerte, lo que fue el omnipotente Estado nacional, desarrollado después de la Segunda Guerra Mundial, ha de ser redefinido y reinventado, probablemente siguiendo directrices similares a las aplicadas por las empresas globales modernas (Pérez, 2001, p. 18).

En este último sentido, recomienda imitar en el sector público las redes del sector privado, haciendo propia de esta forma la configuración organizativa del actual PTE (Pérez, 2001). En realidad, esta apreciación no constituye únicamente una recomendación de política, sino una constatación fundada sobre un principio general que establece cuando piensa la relación entre lo económico y lo institucional durante los cambios de paradigma: "In fact, the common sense principles of organisation for maximum efficiency and effectiveness embodied in the techno-economic paradigm gradually spread out of the business world and into government and other non profit institutions" (Pérez, 2009b).

De tal modo, formando parte de una red,

el Estado nacional central puede ejercer su función de liderazgo orientando las actividades de los distintos agentes sociales para que converjan en una dirección general de cambio convenida de común acuerdo. También puede desempeñar un papel fundamental como intermediario entre actores e instituciones emergentes a nivel global o suprarregional y los agentes regionales, locales e incluso municipales o parroquiales, cuya autonomía tiende a ser cada vez mayor. Se está produciendo también un proceso de difusión del poder. [...] El Estado nacional debe ser capaz de actuar como intermediario dentro del país y entre los diversos niveles supranacionales y subnacionales a fin de promover y negociar oportunidades equitativas para todos. Asumiendo el papel de promotor del consenso entre los distintos protagonistas, es posible que el Estado logre ejercer una autoridad más eficaz, como base para establecer un poder real que le permita influir en el curso de los acontecimientos (Pérez, 2001, p. 19, cursivas del original). 
Pérez denomina a este nuevo tipo de Estado "desarrollista dinámico", cuyo asiento debe ser la administración local, pero orientada a la inserción global. El Estado desarrollista dinámico sería el encargado de promover el consenso entre los actores sociales implicados en los procesos de innovación y desarrollo. Y, para ella el grado de consenso o de conflicto incide de forma determinante tanto en la rapidez como en la facilidad con que puede establecerse el nuevo modo de crecimiento (Pérez, 1997). Al mismo tiempo, el ejercicio de esta función básica permitiría generar las condiciones sociales fundamentales para la consecución de las metas del desarrollo: "avanzar hacia la participación directa de toda la población en actividades generadoras de riqueza” (Pérez, 1997, p. 9).

Llamativo es también que las escasas referencias al rol del Estado en los procesos de desarrollo nunca aparezcan ligadas a las reflexiones sobre la distribución. Es decir que, en apariencia, en la teoría de Pérez el Estado ya no interviene de forma determinante en la distribución de la riqueza. Al menos no explícitamente. Según hemos visto, las funciones propias del Estado se orientan a incidir en la generación de las condiciones sociales para la elevación de la productividad general de la economía y aportar así al incremento de la competitividad internacional.

En efecto, en los escasos pasajes en que esta autora aborda el problema de la distribución, la explicación del fenómeno se basa en alusiones, generales, ambiguas y permanentes, a las relaciones de las fuerzas sociales. La configuración que éstas adquieran en cada fase de evolución del sistema determinará la forma de distribución de la riqueza. Hasta aquí la explicación no resulta para nosotros esencialmente insatisfactoria, ni presenta, en un plano muy general, rasgos tan diferentes a los que podemos encontrar en otros marcos teóricos cuyo poder explicativo resulta ya indiscutible en el campo de las ciencias sociales contemporáneas, más allá de los acuerdos o desacuerdos de perspectiva. En los textos de Pérez, las fuerzas sociales, las instituciones, lo institucional, lo socioinstitucional, tienen un valor explicativo equivalente y cumplen las mismas funciones en su esquema causal. Pero si intentamos penetrar esta primera aproximación e indagar sobre la composición y el carácter específico de las entidades y dimensiones de lo social que nombra diversas maneras, nos encontramos con un espacio vacío.

Hasta aquí hemos llamado la atención sobre dos cuestiones relativas al problema del Estado en la teoría de Pérez: la ausencia de una reflexión teórica profunda sobre su naturaleza y su papel en los procesos de innovación y desarrollo, $\mathrm{y}$, derivado de ello, la ausencia de referencias a la acción estatal en la consideración del problema de la distribución de la riqueza, junto a una asimilación 
del Estado a las instancias socioinstitucionales del sistema que no merece, en la consideración de esta investigadora, mayores argumentos. Ahora bien, entendemos que esta forma de conceptualización, carente de profundidad explicativa, no sólo dificulta una comprensión precisa de lo que son las fuerzas sociales en la visión de Pérez, sino que impide, en particular, interpretar con un grado razonable de certeza si el Estado es o no una fuerza social; si no lo es, pero se articula con ellas al interior del subsistema socioinstitucional, o las representa, etcétera. Además, la ausencia de una reflexión sistemática sobre el Estado en particular y sobre las fuerzas sociales en general impide comprender cuál es su relación con la instancia económica del sistema, considerando que para ella es allí donde se encuentra el factor determinante principal del cambio. En cualquier caso, por lo ya explicado acerca de su noción de las instituciones, queda claro que lo concibe como una instancia sistémica separable (analíticamente) y separada (en el sistema) de la tecnoeconómica. Siendo así, surge la pregunta de cuáles son para Pérez las formas específicas de relación entre el Estado y los agentes económicos, y entre éste y los agentes sociales en general. Para intentar responder esta cuestión tomamos como referencia las funciones -ya expuestas-que la autora le atribuye en términos normativos. Podemos observar tres tipos posibles de relación; los dos primeros referidos específicamente al vínculo con los agentes económicos y el tercero, al vínculo con los agentes sociales en general, en los que entendemos están incluidos los anteriores:

a) Plantea que el Estado no debe proteger a la empresa privada sino dejar que ésta descubra por sí misma las transformaciones del mercado mundial y se decida a emprender su propia modernización. Esto es, que no debe intervenir sobre las condiciones del mercado y las decisiones de la empresa, sino que es ésta la que debe decidir qué rumbo asumir en función de esas condiciones de mercado. Podemos decir entonces que ello implica una concepción autonómica del vínculo entre la instancia estatal y la empresarial.

b) Además, propone al Estado imitar, ante cada cambio de paradigma tecnoeconómico, las redes del sector privado. Es decir, que tiene la tarea de autotransformarse en virtud de las mudanzas organizativas (políticas) que imponen los cambios en el campo empresarial. Ello implica un vínculo que podemos denominar isomórfico entre el Estado y los agentes económicos, por el que el primero se relaciona desde fuera con los segundos y adopta la forma organizativa de ellos. 
c) Por último, postula que el Estado debe orientar las actividades de los distintos agentes sociales para que converjan en una dirección general de cambio convenida de común acuerdo, actuar como intermediario dentro del país y entre los diversos niveles supranacionales y subnacionales a fin de promover y negociar oportunidades equitativas para todos, y promover el consenso entre los distintos protagonistas. Esto implica, para nosotros, un rol de articulación política extrínseca de los actores sociales ajenos al Estado.

Sostenemos que esta forma de entender los vínculos del Estado con los agentes privados implica una concepción restringida del primero -que percibimos a lo largo de toda la producción de Pérez-, en dos sentidos, principalmente:

1) Por una parte, lo concibe como un agente homogéneo. Queremos decir con esto que no reflexiona sobre el Estado como un espacio atravesado, constitutivamente, por conflictos políticos y económicos que ponen en cuestión su propio carácter como institución política y económicamente unificada. Es decir, se plantea que debe generar consenso entre los demás actores, pero el consenso al interior de su propia estructura se supone ya dado. Como inferencia teórica y metodológica, ello impide reflexionar sobre las dificultades intrínsecas del Estado para diseñar estrategias de desarrollo y conducir de modo coherente su implementación en conjunto con el resto de los agentes del sistema. Sobre todo durante periodos de cambio sistémico que, por definición, implican inestabilidad en todos los órdenes.

2) Por otra parte, en todos los casos y en parte por los motivos arriba indicados, el Estado es concebido en un vínculo de exterioridad con el resto de los agentes del sistema sin problematizar, de modo sistemático y en términos positivos, las relaciones múltiples que establece con todos ellos, relaciones que implican a su vez condicionamientos históricos - productivos y restrictivos-a su capacidad de acción para conducir los procesos de desarrollo, sobre todo en los PED. En otras palabras, se atribuye al Estado una capacidad diferencial de liderazgo durante los periodos de cambio de paradigma, sin indagar sobre el resto de los factores de poder que inciden en la definición del rumbo del desarrollo, que han estado a su vez históricamente entrelazados con las estructuras estatales. 
Sin una reflexión sistemática sobre estas cuestiones, resulta difícil analizar profundamente los modos en que los PED pueden procesar internamente los cambios de paradigma e insertarse en un medio económico internacional constitutivamente transformado en relación con los parámetros históricos de producción y distribución de la riqueza a nivel mundial. Por otra parte, es importante reconocer que la de Pérez es una teoría de alcance macroeconómico, por lo cual la presente crítica resulta en sí misma insuficiente como propuesta de análisis del rol del Estado en procesos de desarrollo específicos. Por ello, se considera de interés introducir algunas nociones provenientes de la sociología del desarrollo que pueden ser de ayuda en ese sentido.

\section{Intervención estatal y desarrollo sectorial: los aportes de la sociología del desarrollo}

Algunos antecedentes en la denominada sociología del desarrollo proveen nociones útiles para analizar de modo específico la acción del Estado en relación con los agentes económicos (Schneider y Wolfson, 1999; Viguera, 1998).

En particular, nos referimos a aquellas que explican el carácter de la intervención económica y político-institucional del Estado y su incidencia sobre la acción y el desempeño de los agentes privados.

La intervención estatal destinada a afectar la actividad económica de actores específicos es una variable fundamental para comprender los procesos de crecimiento sectoriales y, por lo tanto, la evolución general de una estructura productiva, cuya orientación se supone siempre determinada en gran medida por la incidencia del Estado en términos macro- y microeconómicos. En este sentido, definimos la intervención económica estatal como el conjunto de sus acciones, identificables en un marco temporal determinado, que dan cuenta de sus funciones regulatorias y productivas. En particular, se trata de las acciones regulatorias de la actividad del mercado y de aquéllas en su carácter de agente económico, es decir, como productor y demandante de los frutos de la actividad productiva, y/o como financiador directo o indirecto de la actividad de los agentes privados. Estas acciones definen el tipo y la orientación de las transferencias de recursos públicos hacia el capital. En virtud de ello, inciden de forma decisiva en el proceso de acumulación de las empresas privadas (Castellani, 2006; Borrastero, 2011).

Al mismo tiempo, la intervención político-institucional del Estado es el conjunto de acciones destinadas a modificar y/o crear arreglos institucionales 
específicos para la definición de políticas públicas, cuya orientación siempre sectorial implica formas determinadas de involucramiento de los agentes privados en la formulación y ejecución de las políticas (véase Borrastero, 2011).

A partir de la intervención estatal y del accionar de los agentes privados se producen formas de articulación público-privada cuyo carácter determinará en gran medida el nivel de desempeño alcanzado por parte de los actores involucrados.

La intervención estatal está siempre presente en cualquier tipo de modelo productivo e institucional que configure la economía nacional. Esto es, su existencia no depende de la voluntad de los gobiernos, sino sólo su orientación específica, que dará forma a su vez al tipo de articulación público-privada, y así, al modelo productivo e institucional vigente.

Estas nociones resultan útiles en términos metodológicos, como variables operativas que permiten analizar casos de desarrollo sectorial, sobre todo aquellos relacionados fuertemente con el accionar estatal. En este sentido, serán fructíferas para encarar un análisis preliminar e ilustrativo del caso del sector de SSI de la ciudad de Córdoba, Argentina, que se ofrece en la siguiente sección, en contraste parcial con los conceptos vertidos por Pérez.

\section{UN ANÁLISIS DE LA INTERVENCIÓN ESTATAL EN EL DESARROLLO DEL SECTOR DE SOFTWARE Y SERVICIOS INFORMÁTICOS DE LA CIUDAD de Córdoba, Argentina}

En primer lugar, el caso es relevante y su análisis pertinente por tres razones principales: se trata, en efecto, del sector donde se produce el factor clave del actual PTE; en segundo lugar, es un sector cuyo desarrollo reciente ha estado profundamente marcado por la acción del Estado, ${ }^{8}$ y por último, la evolución reciente el sector de SSI de la ciudad de Córdoba (capital de la provincia del mismo nombre) muestra altos indicadores de desempeño en relación con las décadas anteriores. ${ }^{9}$

\footnotetext{
${ }^{8} \mathrm{La}$ autora de este artículo ha analizado en otros lugares la intervención del Estado y el desempeño del sector en términos históricos (Borrastero, 2011; Castellani y Borrastero, 2011). Se remite a esos trabajos para ampliar tanto los datos presentados en este apartado como los conceptos vertidos en el anterior.

${ }^{9}$ Además, Córdoba es la segunda ciudad más importante de Argentina (luego de la Capital Federal), en términos de su participación en el PIB argentino y su nivel de desarrollo industrial y urbano, lo cual otorga al caso una relevancia analítica particular.
} 
El sector de SSI cordobés ha crecido en forma constante desde principios de este siglo. A modo de hipótesis, se sostiene que su ritmo de crecimiento acelerado se ha dado a partir de la adopción de una estrategia de promoción diferencial impulsada por el gobierno de la provincia de Córdoba, en el marco de la consolidación del modelo productivo nacional y la implementación de la Ley Nacional de Promoción de la Industria del Software. En particular, se considera que el tipo de articulación público-privada observada en el sector ha generado un proceso relativamente virtuoso, a partir de rasgos diversos a los propuestos por Carlota Pérez: el vínculo establecido entre el Estado y los agentes privados se revela como no autonómico, no isomórfico, ni de articulación política extrínseca. En contraste, la dinámica de dichos vínculos en la última década ha ido adquiriendo paulatinamente un carácter estructural, ha generado nuevas estructuras burocráticas al interior del Estado y la articulación de las políticas se ha dado de manera conjunta.

Para organizar la descripción de la evolución del sector se desarrollarán los siguientes tópicos: primero, la presentación de los datos de desempeño sectorial; luego, una historización del crecimiento del sector en términos de los hitos principales y las acciones de sus actores: el gobierno provincial y los empresarios privados; a continuación se introducirán algunos datos acerca de las políticas del Estado argentino para el sector de SSI, útiles para contextualizar el desarrollo local del sector; por último, una reflexión acerca de la evolución sectorial en términos de las hipótesis planteadas y sus implicaciones para la concepción del Estado de Carlota Pérez.

Los datos de demografía empresarial sistematizados en el cuadro 1 resultan indicativos del crecimiento del sector.

Cuadro 1. Demografía empresarial del sector de ssı de la ciudad de Córdoba, Argentina, 2010

\begin{tabular}{|l|l|}
\hline \multicolumn{1}{|c|}{ Indicador } & 2010 \\
\hline Cantidad de empresas & $92 *$ \\
\hline Empresas que producen software & $78 \%$ \\
\hline Cantidad de empresas que exportan & $55 \% * *$ \\
\hline Empresas asociadas al Cluster Córdoba Technology (CCT) & $78 \%$ \\
\hline
\end{tabular}

* En 2001 eran 30.

** En 2001 representaban el 3\%.

Fuente: Elaboración propia con base en Borrastero (2011). 
En el año 2001 el total de empresas ascendía a 30, es decir, un tercio de la cantidad actual. ${ }^{10} \mathrm{El}$ grupo de las exportadoras representaba en ese año el 3\% del total, mientras que en 2010 ascendía a 55\%. Además, en 2010 era muy alta la proporción de empresas (78\%) que producían software -el segmento de mayor complejidad-, mientras que en 2001 presentaban un nivel tecnológico medio. Por último, en 2010 era alto el grado de asociación institucional observado en el sector: el 78\% de las empresas estaban asociadas al Cluster Córdoba Technology (СCT), entidad civil de promoción de las pymes del sector fundada a fines de 2001.

Los indicadores de desempeño sectorial que se observan en el cuadro 2 también muestran mejorías importantes.

Cuadro 2. Datos de desempeño del sector de ssı de la ciudad de Córdoba, Argentina, 2001-2010

\begin{tabular}{|l|c|c|c|}
\hline \multirow{2}{*}{ Indicador } & 2001 & \multicolumn{2}{c|}{2010} \\
\cline { 2 - 4 } & Pymes locales & Multinacionales** & Pymes locales \\
\hline Facturación* & 27.5 & 62.5 & 100 \\
\hline Exportaciones* & 2.5 & 50 & 17.5 \\
\hline $\begin{array}{l}\text { Cantidad de } \\
\text { empleados }\end{array}$ & 800 & 1500 & 3600 \\
\hline
\end{tabular}

* En millones de dólares estadunidenses.

** No se consignan datos de las empresas multinacionales para 2001 debido a que en ese año todavía no se habían radicado en la ciudad, como se verá más adelante.

Fuente: Elaboración propia en base a datos del Plan Estratégico de Software de la Provincia de Córdoba (Mesa Sectorial TIC, 2010).

Con respecto a los datos de empleo, se observa que en las pymes locales este indicador se ha cuadruplicado a lo largo de la década, al pasar de $800 \mathrm{em}-$ pleados en 2001 a 3600 en 2010. Vale aclarar que el total consignado en el cuadro 2 corresponde exclusivamente a los ingenieros y técnicos empleados en las pymes del sector. Si se suman a ellos los empleados de la administración y tareas anexas, el total de trabajadores llegaba a 10000 en 2010. Según datos de la prensa especializada, el sector de SSI es actualmente uno de los mayores generadores de empleo en la provincia de Córdoba. ${ }^{11}$ Por otra parte, corresponde resaltar que

\footnotetext{
${ }^{10}$ Los datos sobre estos tópicos no contenidos en el cuadro 1 están basados en Borrastero (2011).

${ }^{11}$ Como punto de comparación, un informe de la revista Mercado (s.a., 2008) señala que el sector automotriz -el más desarrollado de la industria local- habría generado hasta 2010 unos 6000 puestos de trabajo.
} 
se trata en términos generales de un sector de alta calificación. Los niveles de facturación, como puede observarse, han crecido significativamente, pasando de 27.5 millones de dólares estadunidenses en 2001 a 100 millones en 2010. Y un último dato llamativo es que, a excepción del monto de exportaciones, el desempeño del sector local en 2010 fue claramente superior al del sector transnacional.

En el cuadro 3 se observan los guarismos para el valor bruto de la producción (VBP) y el valor agregado (VA) del sector entre 2000 y 2009, así como la participación relativa del sector en la estructura del producto bruto geográfico $(\mathrm{PBG})$ de la provincia de Córdoba.

Cuadro 3. Evolución de la participación del sector informático en el PBG de la provincia de Córdoba, 2000-2009

\begin{tabular}{|l|c|c|}
\hline \multicolumn{1}{|c|}{ Indicador } & 2000 & 2009 \\
\hline Valor bruto de producción (VBP)* & 40208 & 54742 \\
\hline Valor agregado (VA)* & 25936 & 35311 \\
\hline Porcentaje del producto bruto geográfico (PBG) & 0.19 & 0.19 \\
\hline
\end{tabular}

* En miles de pesos argentinos constantes de 1993, a precios de productor.

Fuente: Elaboración propia con base en datos del PBG provincial a dos dígitos y la Cuenta de Inversión de la Provincia de Córdoba (2002 y 2009).

En dicho cuadro es posible observar un incremento importante del VBP y el va sectoriales, no así un aumento de la participación en el PBG, que se ha mantenido constante.

Este proceso de crecimiento muestra en la actualidad resultados considerables para el posicionamiento de Córdoba como uno de los polos tecnológicos más importantes de Argentina. Por un lado, se encuentra en proceso la construcción del Parque Empresarial Aeropuerto, ${ }^{12}$ que albergará a las multinacionales radicadas en la ciudad y a las pymes pertenecientes al СCT. Por otro lado, se creó

${ }^{12}$ Se trata de un moderno complejo basado en el concepto de "parque suburbano de oficinas", ubicado en un terreno de 46 hectáreas frente al aeropuerto internacional de Córdoba. La iniciativa surgió de cuatro grandes firmas locales, con el objetivo de reunir a empresas de alto perfil tecnológico, empresas de servicios y grandes emprendimientos comerciales para "generar sinergias positivas" al interior del tejido empresarial de mayor nivel en la región (véase www.parque-empresarial. com). Por convenio con el gobierno provincial, el Banco de Córdoba y las asociaciones del sector de SSI, PEASA, la firma dueña del parque, ha donado varios predios para las empresas y entidades locales vinculadas al sector, y la provincia subsidiará la construcción de los edificios y el pago de los servicios (en su totalidad en el caso de las transnacionales y en forma parcial para las pymes). 
recientemente la Fundación Córdoba TIC, una iniciativa que es hoy el corolario de una historia de institucionalización creciente de los vínculos entre el gobierno povincial y las empresas del sector. La fundación es una entidad mixta (estatalempresarial) creada por ley, ${ }^{13}$ destinada a institucionalizar la participación del sector privado en la definición y ejecución de las políticas sectoriales, con dos propósitos específicos: por un lado, incrementar la capacidad burocrática para el diseño e implementación de las políticas, ganando en dinamismo y relegando parte de la autonomía estatal en la toma de las decisiones estratégicas; por otro lado, compartir la financiación de las acciones sectoriales..$^{14}$ Es éste el nuevo espacio de conducción del sector, conformado por representantes del CCT, la Cámara de Industrias Informáticas, Electrónicas y de Comunicaciones del Centro de Argentina (CIIECA), los ministerios de Industria, de Educación y de Ciencia, Tecnología e Innovación Productiva (Mincyt) ${ }^{15}$ la Agencia Pro Córdoba ${ }^{16}$ y dos universidades: ${ }^{17}$ ocho en total, con igual representación. Los miembros designarán un consejo de administración que manejará planes y fondos, y la presidencia de la fundación queda en manos del sector privado. ${ }^{18}$ La iniciativa surgió a partir de la antigua Mesa Sectorial TIC, un espacio de intercambio no formalizado entre los mismos actores, convocado originalmente por el Ministerio de Industria de la provincia a partir de la demanda del sector empresarial local. Antes de la existencia de la mesa, la política sectorial era conducida por el mencionado ministerio.

En una perspectiva histórica, dos acontecimientos significativos dieron lugar al crecimiento del sector local en la última década: la radicación de Motorola en la ciudad y la fundación del CCT, que agrupa a la gran mayoría de las

\footnotetext{
${ }^{13}$ Se votó en el mes marzo de 2011 en la Legislatura provincial.

${ }^{14} \mathrm{El}$ aporte previsto por parte del sector privado (45\%) supera ligeramente al del sector público (40\%); el restante 15\% del presupuesto sería aportado por las universidades. En total, se prevé un presupuesto anual de 44.5 millones de pesos argentinos entre 2011 y 2020 . El $60 \%$ de dicho presupuesto estaría destinado a la capacitación de recursos humanos -principal carencia del sectora través de planes de becas, incubadoras de empresas y estrategias de internacionalización, entre otras.

${ }^{15}$ El Mincyt de Córdoba fue creado en 2007. Además de la dirección de la política científicotecnológica, tiene a su cargo la relación con el sector productivo, a través de la Secretaría de Vinculación Tecnológica (Secvit), que desde el comienzo generó lazos estrechos con los empresarios del sector de SSI a través del CCT y la CIIECA.

${ }^{16}$ Agencia de promoción de la economía regional.

${ }^{17}$ La Universidad Nacional de Córdoba y la Universidad Tecnológica Nacional-Regional Córdoba.

${ }^{18}$ Hasta el momento no ha sido oficializado, pero en la prensa especializada se descuenta que la presidencia del ente estará a cargo del sector privado.
} 
pymes locales de software. ${ }^{19}$ Lo primero se dio a partir de intensas negociaciones de Motorola con el gobierno provincial de Córdoba, que competía con Rosario, para concretar las intenciones de esa empresa de instalarse en Argentina. En efecto, los exorbitantes beneficios otorgados a Motorola implicaron una inversión estatal de gran envergadura que continúa hasta hoy: ${ }^{20}$ la construcción de edificios totalmente equipados, el pago de alquileres durante años, el subsidio del 30\% de los gastos salariales, la exención impositiva total de los tributos provinciales, etcétera

La creación del СCT fue una iniciativa de un grupo de empresarios locales, en vistas del potencial y la amenaza que al mismo tiempo significaba la radicación de Motorola, y de la firmeza que exhibía el discurso del gobierno provincial en cuanto a su intención de promover el desarrollo del sector informático.

El proceso de desarrollo del sector local, en toda su complejidad, ha sido posible a su vez en el marco de la evolución general del sector de SSI a nivel nacional, impulsada por el Estado argentino. De hecho, en los últimos años, la industria de SSI ha comenzado a ocupar un lugar destacado en la estructura productiva del país. Este cambio se debe en gran medida al fuerte impulso de la política tecnológica nacional de la última década, del cual distinguimos dos ciclos: el primero corresponde al periodo 1999-2004, y el segundo va desde 2004 (año en que se sancionó la Ley Nacional de Promoción de la Industria del Software) hasta la actualidad. ${ }^{21}$ En la década de los setenta, Argentina ya contaba con una oferta local de software, aunque muy incipiente. A mediados de los ochenta, el mercado había crecido cuatro veces en su nivel de facturación, aunque en otros aspectos no se habían producido avances de importancia: el 70\% de ese mercado era abastecido aún por productos extranjeros, el grado de concentración de la industria era muy elevado, las exportaciones eran ocasionales y los desarrollos se vinculaban casi exclusivamente a aplicaciones administrativas a medida. Hacia fines de los noventa, el sector facturaba unos 190 millones de dólares y empleaba en total a unas 4500 personas, ${ }^{22}$ pero no presentaba un crecimiento considerable

\footnotetext{
${ }^{19}$ Además, ese mismo año se creó la CIIECA, que reúne a las empresas electrónicas, otro sector industrial de cierta relevancia en el tejido productivo de la ciudad. Desde entonces, el CCT y la CIIECA impulsan conjuntamente la gran mayoría de las iniciativas de interés sectorial.

${ }^{20}$ Véase Borrastero (2011).

${ }^{21}$ El desarrollo de este tópico se basó en Borrastero (2011).

${ }^{22}$ El salto en este último sentido se produjo particularmente en el área de desarrollo de software, que pasó de 1180 empleados aproximadamente en la década de los ochenta, a unos 3000 en los noventa. El área de servicios informáticos, en cambio, experimentó un descenso durante el mismo periodo, pasando de 2000 a 1500 personas.
} 
en términos de cantidad de firmas activas, expansión del mercado de desarrollo y niveles de exportación. En 2000, se observaron niveles de facturación superiores, junto a un leve descenso de la participación de las firmas extranjeras sobre el total facturado y una ampliación en cuatro puntos de la porción generada por las empresas nacionales. El empleo tuvo una baja de 1000 puestos con respecto a la década de los noventa entre las firmas locales, que no obstante mantuvieron un nivel de participación mayor al de las transnacionales. ${ }^{23}$ En cuanto a las exportaciones, las empresas extranjeras lideraban claramente la actividad, con el $73 \%$ del total. Hacia el año 2001, se estimaba que el sector tenía ya una importancia significativa en la industria nacional y que continuaría expandiéndose si se consolidaban tres tendencias ya incipientes en el periodo: una mayor disponibilidad de recursos humanos calificados, el avance en la calidad de las telecomunicaciones del país y un tipo de cambio favorable a la exportación y la competitividad salarial. Dichas tendencias se mantuvieron durante los años posteriores a la devaluación del peso en 2002, como producto de la política económica y tecnológica asumida por el gobierno argentino; la sanción de la Ley Nacional de Promoción de la Industria del Software en 2004 vino a profundizar las condiciones favorables para el sector mediante normas impositivas especiales. Dicha ley considera por primera vez la producción de software como una actividad industrial y la sujeta a los beneficios especiales de los que ésta goza en Argentina: exención por diez años de los impuestos industriales; crédito fiscal para cancelar IVA y otros impuestos; desgravación del $60 \%$ sobre el impuesto a las ganancias para quienes acrediten investigación y desarrollo, certificación de calidad y/o exportaciones de software; desgravación de la importación de hardware para la producción. ${ }^{24}$ De modo que, a diciembre de 2009, el sector nacional facturaba unos $3000 \mathrm{mi}$ llones de dólares, contaba con unos 56700 puestos de trabajo directos y las exportaciones representaban más de 600 millones de dólares.

Estos datos contextuales acerca de la evolución general de la industria argentina del software resultan ilustrativos del impulso del que gozó el sector a partir del fin del régimen de convertibilidad de la moneda encarado por el Estado argentino, que tuvo un impacto significativo en todas las regiones productoras de software del país, entre las que Córdoba ocupa el segundo lugar en importancia, luego de la región metropolitana de Buenos Aires. En este marco, como se vio

\footnotetext{
${ }^{23}$ También bastante elevado: 42\%, con 2792 personas.

${ }^{24}$ Ley nacional No. 25922, sancionada el 18 de agosto de 2004. Recuperado de: www.agencia. gov.ar. Para ahondar en las políticas públicas nacionales dirigidas al sector de SSI argentino después del fin de la convertibilidad, puede verse Gajst (2011).
} 
antes, el gobierno provincial de Córdoba encaró acciones específicamente destinadas al aprovechamiento local de los recursos puestos a disposición por el Estado, además de implementar sus propios instrumentos de promoción.

En suma, se constata que a partir del año 2001 el crecimiento del sector de SSI de Córdoba ha estado profundamente marcado por ciertos rasgos propios del tipo de intervención económica que la provincia ha definido para impulsar su desarrollo:

a) La radicación de cinco de las multinacionales tecnológicas más grandes del mundo, a partir de la transferencia a estas empresas de cuantiosos recursos financieros estatales, en forma directa (a través de subsidios al empleo e inversión directa: donación de edificios y terrenos, pago de servicios) y en forma indirecta (a través de exenciones impositivas y puesta a disposición de infraestructura física y tecnológica y de recursos humanos).

b) La transferencia a las pymes locales de una cantidad de recursos menor al de los destinados a las transnacionales pero importante en términos históricos y significativa para las empresas del sector local, cuya capacidad de inversión era ciertamente reducida: en forma directa (mediante subsidios al empleo y la instalación de infraestructura física, y políticas de crédito del Banco de la Provincia de Córdoba) e indirecta (sumando apoyos específicos a las capacidades comerciales de estas empresas: capacitaciones, participación en rondas comerciales, integración a programas de promoción, etcétera).

Al mismo tiempo, cuatro rasgos han caracterizado la intervención político-institucional del gobierno provincial:

a) El Estado ha habilitado la posibilidad de establecer y consolidar con el tiempo una política sectorial y, por lo tanto, específica, definida como estratégica.

b) Muchas de las políticas sectoriales han constituido iniciativas propias y muchas otras han surgido como respuesta a las crecientes demandas del sector privado (transnacional y local), ante las cuales el Estado se ha mostrado especialmente permeable.

c) El Estado ha estimulado la incorporación de nuevos actores antes alejados de la definición de la política industrial (por ejemplo, las universidades) 
y ha habilitado canales formales de vinculación antes inexistentes entre los actores no gubernamentales. Hasta el momento de creación de la Mesa Sectorial TIC, las relaciones entre las empresas extranjeras y las locales, y entre las empresas y las universidades eran de carácter informal.

Este artículo sostiene que a raíz de este tipo de accionar se han producido en la provicia transformaciones importantes en las formas de ejercer el liderazgo estatal en el proceso de desarrollo sectorial. Las principales transformaciones que observamos han ocurrido a nivel de:

a) La estructura del gobierno provincial: la creación de nuevos arreglos institucionales destinados específicamente a la definición y ejecución de la política sectorial ha modificado la configuración de los espacios de toma de decisiones. La provincia tiene hoy un nuevo ministerio (de Ciencia, Tecnología e Innovación Productiva), el Ministerio de Industria, Trabajo y Comercio ha asumido nuevas funciones, y se ha formalizado la participación del sector privado en la toma de decisiones estratégicas (por ejemplo, la creación de la mesa sectorial y el paso a la fundación mixta, sancionada con fuerza de ley).

b) Sus funciones específicas: el gobierno provincial no detenta en forma exclusiva la conducción de la política sectorial, sino que la comparte con los actores del sistema económico. En este sentido, podemos afirmar que ha materializado en forma novedosa su función catalizadora de tres fuerzas principales: la presión de los flujos globales de capital, las demandas del sector privado local y la propia necesidad de impulsar el crecimiento económico regional. ${ }^{25}$

c) La naturaleza de los vínculos de la provincia con el sector privado.

d) Las formas de institucionalización de esos vínculos: como puede suponerse, siempre existieron vínculos informales o con diversos grados de formalización con el sector privado, y éstos fueron determinantes para la dirección que asumió el desarrollo del sector a lo largo de la década. Lo que resulta novedoso es el carácter estructural que han ido adquiriendo esos vínculos en los últimos años, según la tendencia observada. Dicho

\footnotetext{
${ }^{25}$ Agreguemos que, al momento de la llegada de Motorola, la tasa de desempleo local venía en aumento desde hacía tres años y las proyecciones al respecto no eran alentadoras. Entre 2001 y 2002 , el desempleo pasó de $12.7 \%$ a $25.3 \%$ respectivamente, y a principios de 2002 la tasa de desocupación había crecido cinco puntos más en Córdoba que el promedio del resto de los conglomerados del país.
} 
carácter se funda sobre dos cambios importantes. Por un lado, los espacios de definición conjunta de políticas no sólo se formalizaron sino que se legalizaron, así como casi todas las acciones relativas al sector (los contratos con las empresas se sancionaron con fuerza de ley, al igual que los planes estratégicos, etcétera). Aquí es muy importante tener en cuenta que las iniciativas de legalización no han estado exentas de conflictos, sino que han sido producto de una larga serie de intensas y problemáticas negociaciones, expresadas sobre todo al interior de los cuerpos legislativos, ${ }^{26}$ en el marco de las fuertes y diversas presiones de los actores del sector privado. Y, por otro lado, mediante los nuevos arreglos institucionales, las relaciones no se han establecido únicamente en forma de "prácticas institucionalizadas" a través de las cuales el sector privado se vincula con la provincia como actor foráneo e independiente, ${ }^{27}$ sino que los agentes privados han pasado a integrar la dirección de la política en materia de SSI.

En virtud de los procesos mencionados, se constata que el tipo de vínculo establecido entre el Estado y los actores privados no ha asumido en este caso los rasgos descritos por Pérez en sus reflexiones sobre el rol estatal en los procesos de cambio estructural y desarrollo. En primer lugar, dichos vínculos no se han revelado como de tipo autonómico, es decir, el Estado no ha dejado a las empresas libradas a las condiciones de un mercado imprevisible. Por el contrario (en particular en relación con el Estado argentino), su intervención económica ha configurado fuertemente las condiciones del mercado que han permitido a las firmas crecer, sobre todo en relación al empleo, al incremento del nivel tecnoló-

${ }^{26}$ Algunos ejemplos entre muchos: cuando Motorola anunció su plan de instalarse en el país, el entonces gobernador de Córdoba, José M. de La Sota, elaboró junto al intendente de la ciudad, Germán Kammerath, una propuesta para la empresa que implicaba "mejorar cualquier oferta" (Da Porta, 2012), lo que generó un conflicto breve pero intenso con la oposición, principalmente porque el convenio previsto significaría para las cuentas de la provincia una erogación de 14 millones de pesos argentinos sólo durante los tres primeros años. Finalmente, el oficialismo logró imponer sus condiciones y la empresa accedió a beneficios exorbitantes de los que goza hasta hoy. Un año después (en 2001), cuando los ejecutivos provincial y municipal acordaron con la misma empresa construir dos edificios para sus instalaciones en un gran predio de propiedad estatal en una zona céntrica (ex-Batallón 141) y donárselos junto con el terreno, la oposición en el Concejo Deliberante de la ciudad lo impidió, en el marco de un intenso conflicto con el intendente. A partir de allí el convenio se modificó en numerosas oportunidades hasta que surgió la posibilidad de construir los edificios en el Parque Empresarial Aeropuerto, recién en 2009.

${ }^{27}$ Véase Dossi y Lissin (2010). 
gico y a la capacidad exportadora. En este sentido, el Estado ha incidido de manera determinante en el proceso de acumulación de las empresas privadas. En segundo lugar, sus vínculos tampoco se han revelado como de tipo isomórfico, esto es, la organización estatal no ha adoptado la forma flexible de las empresas globales a las que ha asistido. En cambio, su intervención político-institucional ha generado nuevas estructuras burocráticas, algunas propias y otras conjuntas, que lejos de obstaculizar el proceso de desarrollo sectorial, lo han permitido y estimulado. Por último, el Estado no ha jugado un rol de articulación política extrínseca, en el sentido de articular desde fuera las acciones de otros actores, oficiando como árbitro de sus interacciones, sino que ha creado espacios de fusión de intereses en torno a la política sectorial en los que él mismo ha sido promotor de una orientación política determinada.

Un aspecto en el que se podría afirmar que se han presentado los rasgos de la acción estatal definidos por Pérez, es el de su homogeneidad y coherencia. Si bien se han suscitado conflictos entre distintas esferas estatales -como hemos visto- el gobierno provincial se ha conducido de forma integrada en la definición e implementación de las políticas sectoriales.

Por último, es posible decir que el tipo de articulación público-privada establecida, en la que el gobierno provincial se ha mostrado especialmente permeable a las demandas del sector privado, no acusa una relación de exterioridad entre la esfera estatal y la empresarial. En contraste, esta última ha incidido en gran medida en la capacidad del Estado para obtener resultados a partir del desempeño de las empresas, sobre todo en relación con las transnacionales, a las cuales ha debido otorgar grandes beneficios sin obtener necesariamente los resultados esperados en cuanto a generación de empleo y captación de rentas fiscales en ese subsector. ${ }^{28}$

No se pretende insinuar con esta crítica que la teoría de Pérez puede ser refutada a partir de las nociones y el análisis de caso presentados. En efecto, sería equívoco considerar que una teoría macroeconómica como la suya, que en principio se propone explicar los ciclos largos de la economía en función de las rupturas tecnológicas, puede ser puesta a prueba de modo completamente imparcial a través del análisis de una región específica en un periodo acotado a una década. Lo que interesa señalar es que si se intentara analizar los roles históricos

\footnotetext{
${ }^{28}$ Líneas de análisis que teorizan especialmente sobre la naturaleza y el rol del Estado en los procesos de desarrollo, y que presentan claves de explicación importantes para un análisis de esta cuestión en los PED pueden encontrarse, por ejemplo, en Amsden (1992), Evans (1995), Skocpol (1985).
} 
del Estado en procesos de desarrollo concretos, entonces se encontraría el tipo de dificultades teóricas mencionadas, que no podrían subsanarse en el marco del estado actual de la teoría con respecto a este tópico. ${ }^{29}$

Queda pendiente una larga serie de tareas en relación con el análisis propuesto, imposibles de realizar en el marco del presente texto. Por una parte, investigar cuáles son los beneficios sociales generalizables del desarrollo del sector estudiado que, como hemos visto, ha implicado una transferencia cuantiosa y permanente de recursos públicos, especialmente hacia el sector transnacional. Por otra parte, indagar más profundamente sobre las posibilidades y los límites de la acción estatal para guiar los procesos de innovación y desarrollo en el marco de una economía globalizada. En relación con ello, es importante tener en cuenta una consideración de Pérez sobre la dinámica global de los PED, a la cual la autora de este artículo se adhiere: los PED son adoptantes del paradigma y no generadores, lo que tiene implicaciones de envergadura para pensar el rol del Estado, en tanto la adopción requiere una adaptación tecnológica, económica, institucional y social partiendo de condiciones que se encuentran desfasadas con respecto al marco que posibilitó el surgimiento y difusión del paradigma original. Por último, es de considerarse esencial la indagación sobre el papel específico que le cabe a la acción estatal en la distribución de la riqueza generada por el desarrollo de las industrias nacionales basadas en el conocimiento. Tareas semejantes no resultan del todo posibles a partir de la teoría evolucionista que se ha analizado, sino que requieren complementar sus aportes con otras nociones que focalicen los roles del Estado en el desarrollo, ampliando su potencial explicativo hacia una comprensión profunda de las particulares condiciones del desarrollo en los PED.

\section{REFERENCIAS BIBLIOGRÁFICAS}

Amsden, A. (1992), A Theory of Government Intervention in Late Industrialization, en Putterman, L., y Rueschemeyer, D. (ed.), The State and Market in Development: Sinergy or Rivalry?, Boulder, Lynn Rienner.

Borrastero, C. (2011), "Intervención estatal, transformaciones en los vínculos con el sector privado y crecimiento económico sectorial. El caso del sector de software

\footnotetext{
${ }^{29} \mathrm{Si}$ se tomara en consideración, por ejemplo, el desarrollo de los países asiáticos para analizarlo desde la teoría de Carlota Pérez, se encontrarían quizás los mismos obstáculos (el grado de intervencionismo del Estado fue allí en general muy alto, y generó nuevas y grandes estructuras burocráticas que permitieron el desarrollo, etcétera; véase, por ejemplo, Amsden, 1992; Evans, 1998; Castells, 1988b).
} 
y servicios informáticos de la ciudad de Córdoba. 2000-2010", H-Industria, Año 5, No. 8. Recuperado de: http://www.hindustria.com.ar/images/client_gallery//HindustriaNro8Borrastero.pdf. Consultado el 27 de septiembre de 2011.

Boyer, R. (1999), "Dos desafíos para el siglo XXI: disciplinar las finanzas y organizar la internacionalización”, Revista de la CEPAL, No. 69, pp. 33-51.

Castellani, A. (2006), Estado, empresas y empresario, la relación entre intervención económica estatal, difusión de ámbitos privilegiados de acumulación y desempeño de las grandes firmas privadas. Argentina 1966-1989, tesis de Doctorado en Ciencias Sociales, Buenos Aires, Universidad de Buenos Aires.

—, y Borrastero, C. (2011), “¿Estrategia o privilegios? Estado y empresarios en el desarrollo del sector software y servicios informáticos de la ciudad de Córdoba (2000-2010)", ponencia presentada en las IX Jornadas de Sociología de la UBA, Buenos Aires, 8 al 12 de agosto de 2011.

Castells, M. (1996), La era de la información. Vol.1. La sociedad red, 2da. ed., Madrid, Ed. Alianza.

- (1988a), Nuevas tecnologías, economía y sociedad, Madrid, Ed. Graymo. (1988b), "The Developmental City-state in an Open World Economy: the Singapore Experience", Center for Advances Studies, Working Paper No. 31, National University of Singapore.

"Cuenta de Inversión de la Provincia de Córdoba" (2002), publicación oficial del ejercicio financiero del Estado Provincial, Contaduría General de la Provincia, Secretaría de Administración Financiera Ministerio de Finanzas de la Provincia de Cordoba. Recuperado de: www.cba.gov.ar. Consultado el 20 de septiembre de 2011.

(2009), publicación oficial del ejercicio financiero del Estado Provincial, Contaduría General de la Provincia, Secretaría de Administración Financiera, Ministerio de Finanzas de la Provincia de Córdoba. Recuperado de: www.cba.gov.ar. Consultado el 20 de septiembre de 2011.

Da Porta, Magdalena (2012, 2 de noviembre), "Reflotan los planes por las sedes de Motorola e Intel", La Voz del Interior, sección de negocios. Recuperado de: http:// www.lavoz.com.ar/noticias/negocios/reflotan-los-planes-por-las-sedes-de-motorola-e-intel.

Dirección General de Estadística y Censos (s.f.), "Producto Geográfico Bruto de la Provincia de Córdoba". Recuperado de: http://estadistica.cba.gov.ar/Econom\%C3\% $\mathrm{ADa} /$ Econom\%C3\%ADayFinanzas/ProductoGeogr\%C3\%A1 ficoBruto/tabid/154/language/es-AR/Default.aspx. Consultado el 15 de agosto de 2011.

Dosi, G. (2003), "Paradigmas tecnológicos y trayectorias tecnológicas. La dirección 
y los determinantes del cambio tecnológico y la transformación de la economía", en Neffa, J., y Chesnais, F. (comp.), Ciencia, tecnología y crecimiento económico, Buenos Aires, Ed. Trabajo y Sociedad, pp. 99-128.

—_ Pavitt, K., y Soete, L. (1990), The Economic of Technical Change and International Trade, London, Harvester Wheatsheaf Press/New York University Press.

Dossi, M., y Lissin, L. (2010), "La acción corporativa: propuesta de abordaje para el estudio del empresariado", ponencia presentada en las V Jornadas de Estudios Sociales de la Economía, IDAES-UNSAM, Buenos Aires, 17, 18 y 19 de noviembre de 2010.

Evans, P. (1995), Embedded Autonomy: States and Industrial Transformation, Princeton, Princeton University Press.

_ (1998), "Alternativas al Estado desarrollista. Lecciones de la crisis de Asia oriental", Nueva Sociedad, No. 155, pp. 142-156.

Gajst, N. (2011), "Las políticas públicas dirigidas a la industria del software y servicios informáticos argentinos en la posconvertibilidad: una historia de la cuestión", ponencia presentada en las IX Jornadas de Sociología de la UBA, Buenos Aires, 8 al 12 de agosto de 2011.

Jessop, B. (2000), "The State and the Contradictions of the Knowledge-Driven Economy”, en Bryson, J.R., et al. (eds.), Knowledge, Space, Economy, London, Routledge, pp. 63-78.

Mesa Sectorial TIC (2010), Plan Estratégico de Software 2010-2020, Córdoba.

Nelson, R., y Winter, S. (1982), An evolutionary theory of economic change, Cambridge, Harvard University Press.

Pérez, C. (1985), "Cambio estructural y asimilación de nuevas tecnologías en el sistema económico y social", Recuperado de: www.carlotaperez.org. Consultado en agosto de 2011.

(1986), "Las nuevas tecnologías: una visión de conjunto". Recuperado de: www.carlotaperez.org. Consultado en agosto de 2011.

_ (1997), "El reto sociopolítico del cambio de paradigma tecnoeconómico". Recuperado de: www.carlotaperez.org. Consultado en agosto de 2011.

_ (1998), "Desafíos sociales y políticos del cambio de paradigma tecnológico", Recuperado de: www.carlotaperez.org. Consultado en agosto de 2011.

(2000), "Cambio de paradigma y rol de la tecnología en el desarrollo". Recuperado de: www.carlotaperez.org. Consultado en agosto de 2011.

(2001) "Cambio tecnológico y oportunidades de desarrollo como blanco móvil", Revista de la CEPAL, No. 75, pp. 115-136.

_ (2004), "Technological Revolutions, Paradigm Shifts and Socio-institutional 
Change", en Reinert, E. (ed.), Globalization, Economic Development and Inequality: An Alternative Perspective, Cheltenham/Northampton, Edward Elgar, pp. 217-242.

_ (2009a), "Technological revolutions and techno-economic paradigms", Working Papers in Technology Governance and Economic Dynamics, Working Paper No. 20, The Other Canon Foundation/ Tallinn University of Technology.

_ (2009b), "La otra globalización: los retos del colapso financiero", Problemas del Desarrollo, Vol. 40, No. 157, 11-37.

s.a. (2008, mayo), “Soja, ruedas, ladrillos y bytes”, Mercado, sección Análisis. Recuperado de: http://www.mercado.com.ar/archivo_nota_revista.php?id=2\&id_edicion $=1082$. Consultado el 10 de agosto de 2011.

Schneider, B. R., y Wolfson, L. (1999), "Las relaciones entre el Estado y las empresas y sus consecuencias para el desarrollo: una revisión de la literatura reciente", Desarrollo Económico, Vol. 39, No. 153, pp. 45-75.

Schumpeter, J. A. (1957), Teoría del desenvolvimiento económico, Distrito Federal, FCE.

Skocpol, T. (comp.) (1985), Bringing the State Back in, Cambridge, Cambridge University Press.

Viguera, A. (1998), "Estado, empresarios y reformas económicas: en busca de una perspectiva analítica integradora", Perfiles Latinoamericanos, Vol. 7, No. 12, México.

Witt, U. (2008), “What is specific about evolutionary economics?", Journal of Evolutionary Economics, No. 18, pp. 547-475. 